Supporting Information (SI)

\title{
Anisotropic Nano-/Microparticles from Diversified Copolymers by Solvent-Mediated Self-Assembly
}

Bin Wang, $\uparrow$ Lingyun Jia, $\ddagger$ Fawei Wang, $\uparrow$ Yufeng He, ${ }^{*} \dagger$ Pengfei Song $\dagger$ and Rongmin Wang ${ }^{*} \dagger$

tKey Laboratory of Eco-functional Polymer Materials of Ministry of Education, Institute of Polymer, College of Chemistry \& Chemical Engineering, $¥$ College of Life Sciences, Northwest Normal University, Lanzhou 730070 , China. *Corresponding author: Tel/Fax.: +86-931-7970358; E-mail: wangrm@nwnu.edu.cn

The Supporting Information (SI) file is of table and figures, 1 table and 6 figures:

$\diamond \quad$ Table S1. Recipe for synthesis of StAPs-A and its comparative experiments.

$\diamond \quad$ Figure S1. Digital photos of different emulsions.

$\diamond \quad$ Figure S2. SEM images of StAPs-2a.

$\diamond \quad$ Figure S3. SEM images of StAPs-a, StAPs-b, StAPs-c, StAPs-d, StAPs-e and StAPs-f.

$\diamond \quad$ Figure S4. SEM images of StAPs-1w and StAPs-2w.

$\diamond \quad$ Figure S5. FT-IR spectra of StAPs.

$\diamond \quad$ Figure S6. ${ }^{1} \mathrm{H}-\mathrm{NMR}$ spectra of StAPs-A. 
Table S1. Recipe for synthesis of StAPs-A and its comparative experiments.

\begin{tabular}{|c|c|c|c|c|c|c|c|}
\hline \multirow{3}{*}{ No. } & \multicolumn{4}{|c|}{ Step 1} & \multicolumn{3}{|c|}{ Step 2} \\
\hline & MAA & MMA & BA & $\mathrm{EtOH} / \mathrm{H}_{2} \mathrm{O}$ & St & BA & PVP (\%) \\
\hline & (g) & (g) & (g) & $(\mathrm{mL} / \mathrm{mL})$ & (g) & (g) & $/ \mathrm{H}_{2} \mathrm{O}(\mathrm{mL})$ \\
\hline StAPs-A & 0.75 & 1.25 & 1.00 & $30 / 10$ & 1.67 & 0.33 & $0.15 / 20$ \\
\hline StAPs-a & I & 1.25 & 1.00 & $30 / 10$ & 1.67 & 0.33 & $0.15 / 20$ \\
\hline StAPs-b & 0.75 & I & 1.00 & $30 / 10$ & 1.67 & 0.33 & $0.15 / 20$ \\
\hline StAPs-c & I & I & 1.00 & $30 / 10$ & 1.67 & 0.33 & $0.15 / 20$ \\
\hline StAPs-d & I & 1.25 & I & $30 / 10$ & 1.67 & 0.33 & $0.15 / 20$ \\
\hline StAPs-e & 0.75 & 1.25 & 1.00 & $30 / 10$ & 1.67 & I & $0.15 / 20$ \\
\hline StAPs-f & 0.75 & 1.25 & 1.00 & $30 / 10$ & I & 0.33 & $0.15 / 20$ \\
\hline
\end{tabular}

Cond: Ms: MAA/MMA/BA=0.75/1.25/1.00

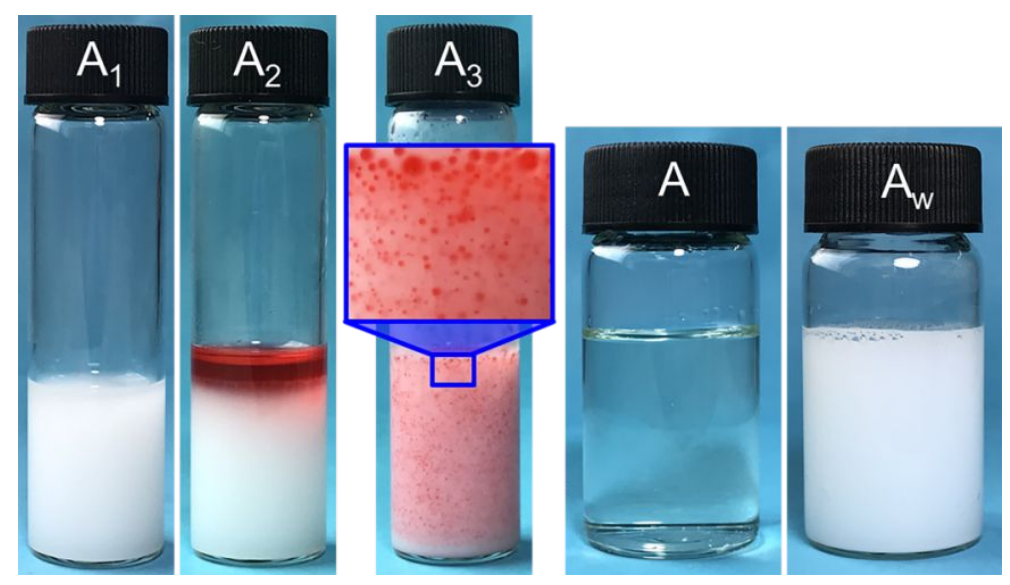

Figure S1. Digital photos of different emulsions (StAPs seed emulsion $\left(A_{1}\right)$ with adding quantitative monomers (St, BA) and marked by Sudan III $\left(A_{2}\right)$, and being fully shaked $\left(A_{3}\right)$; StAPs seed solution (A); StAPs-1w seed emulsion $\left.\left(A_{w}\right)\right)$. 


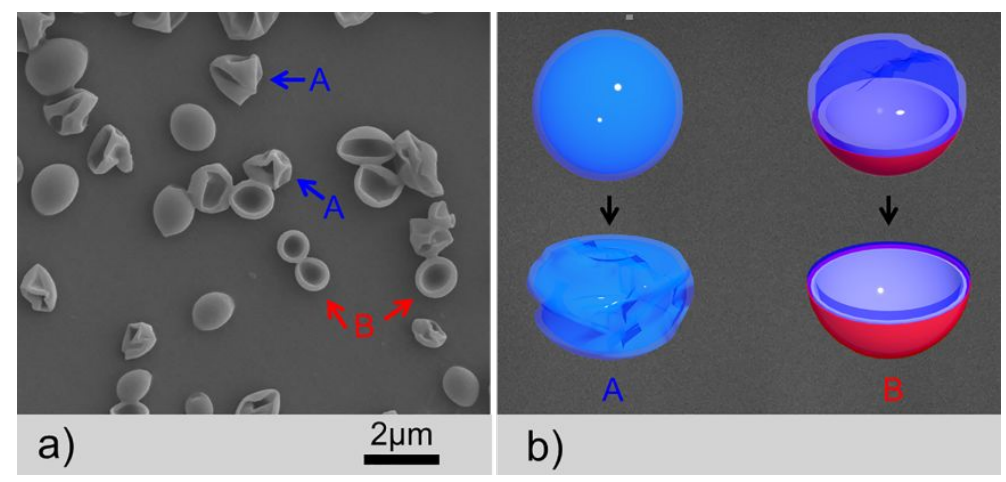

Figure S2. SEM images of StAPs-2a, which is the upper centrifugation separated from StAPs-2 (a); The formation process of StAPs-2a with different morphologies (b). 

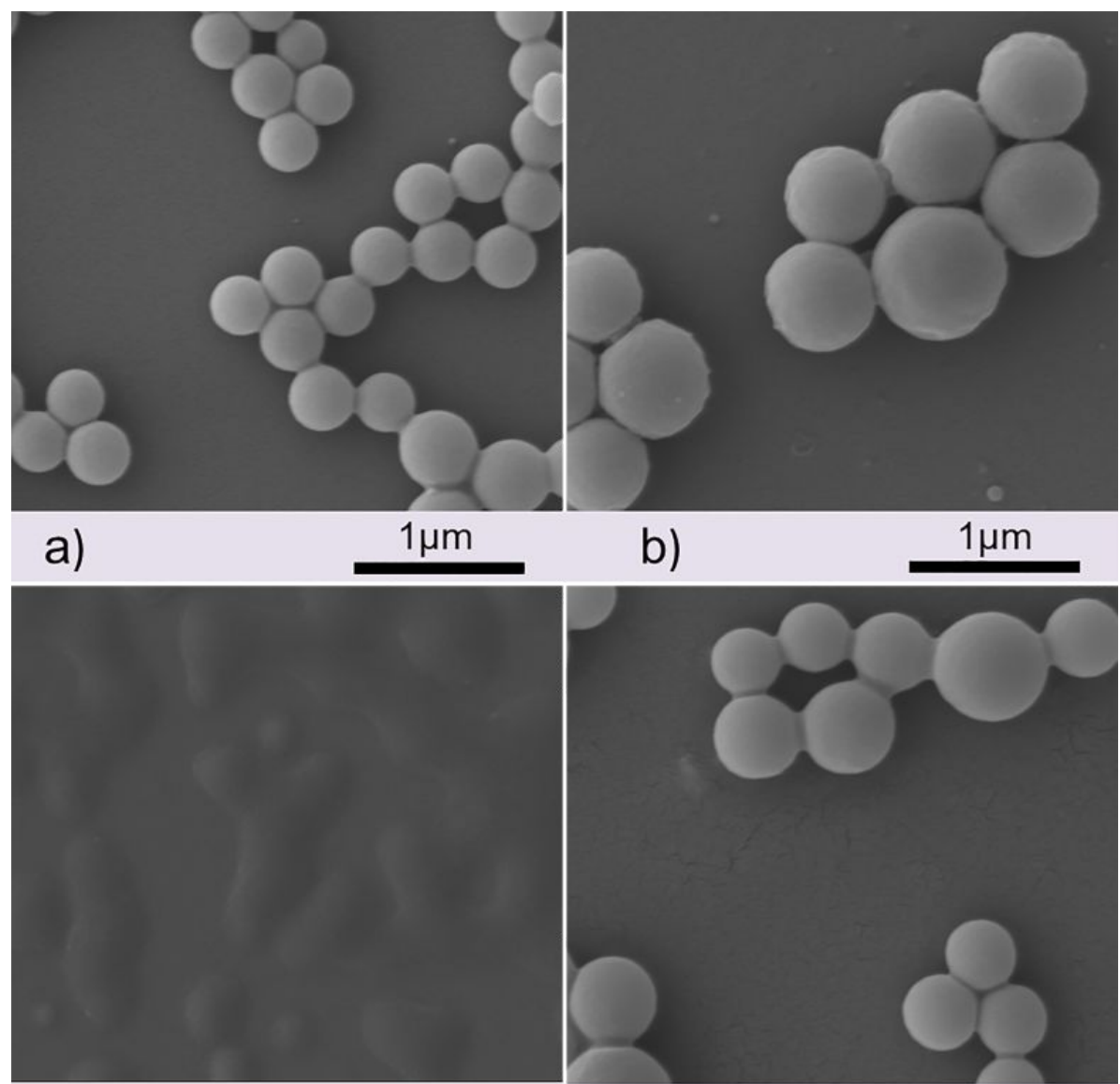

b)

$1 \mu \mathrm{m}$

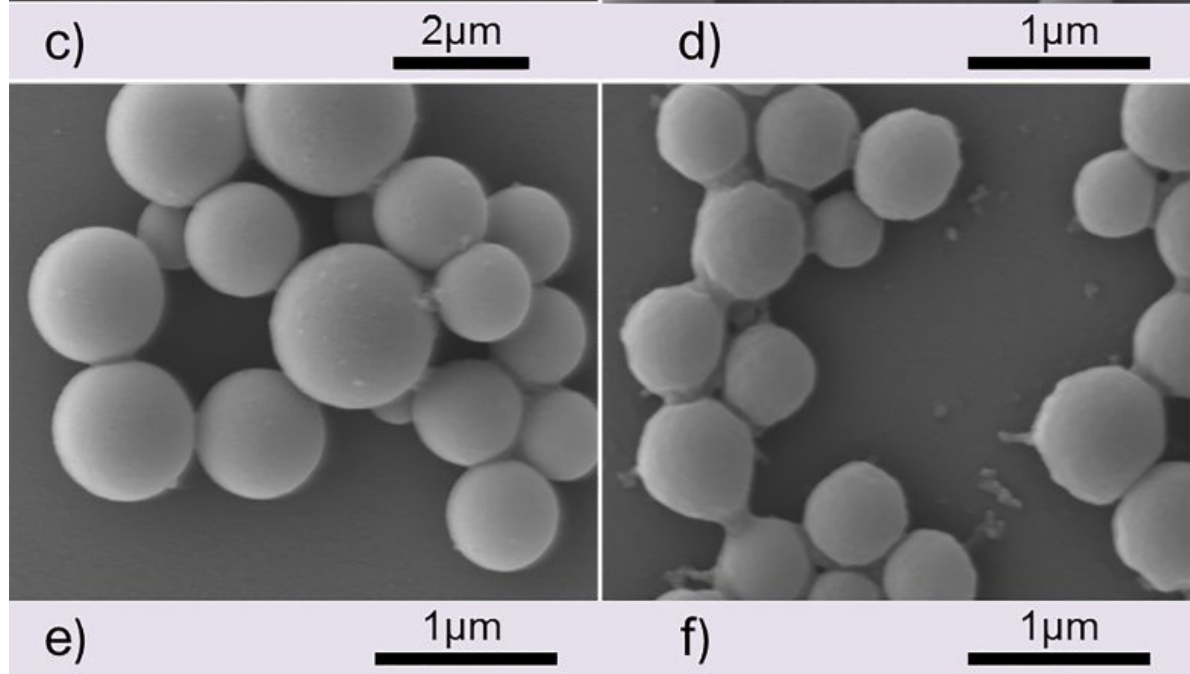

Figure S3. SEM images of StAPs-a (a), StAPs-b (b), StAPs-c (c), StAPs-d (d), StAPs-e (e) and StAPs-f (f). 


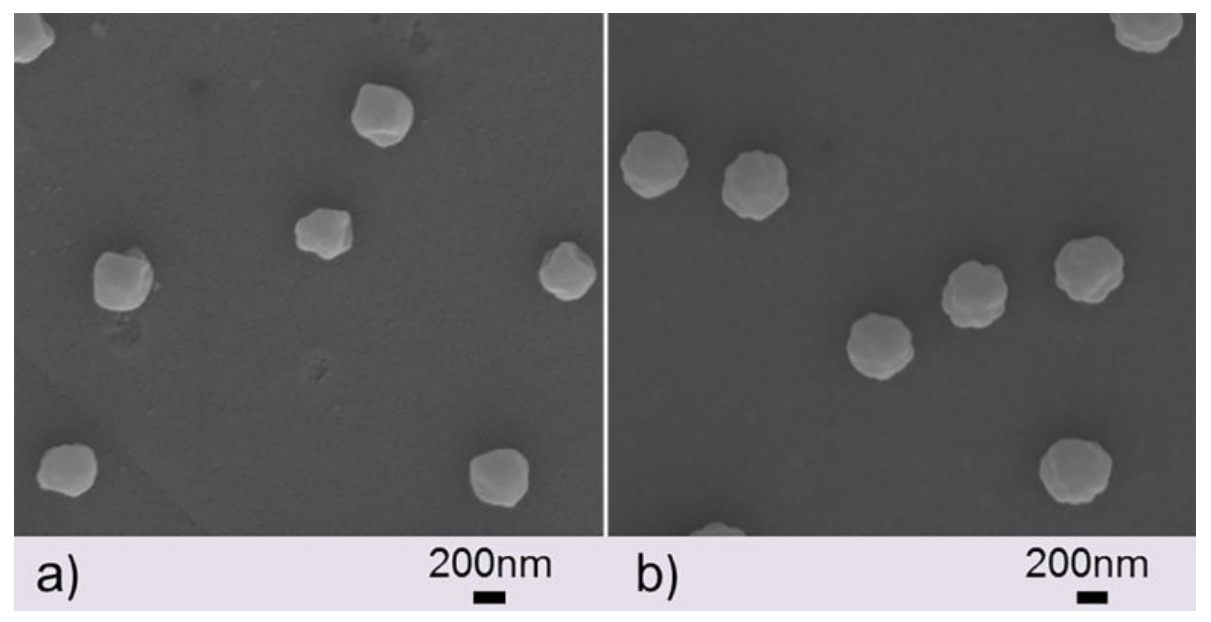

Figure S4. SEM Images of StAPs-1w (a) and StAPs-2w (b).

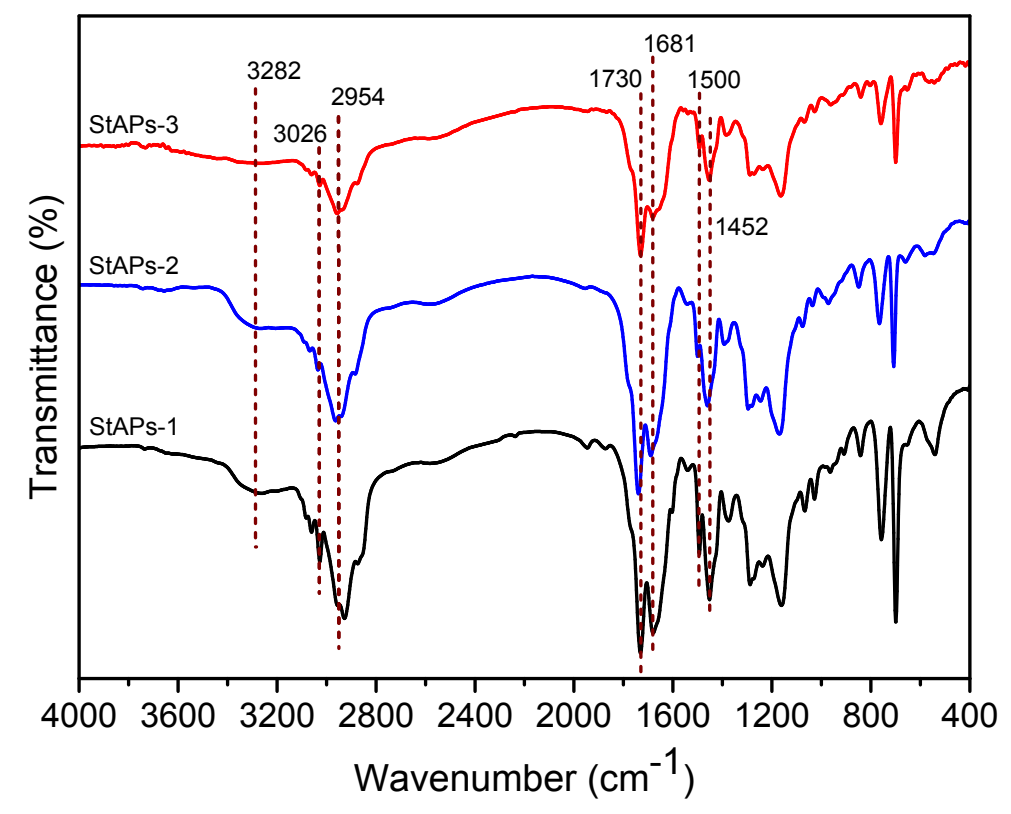

Figure S5. FT-IR spectra of StAPs. 


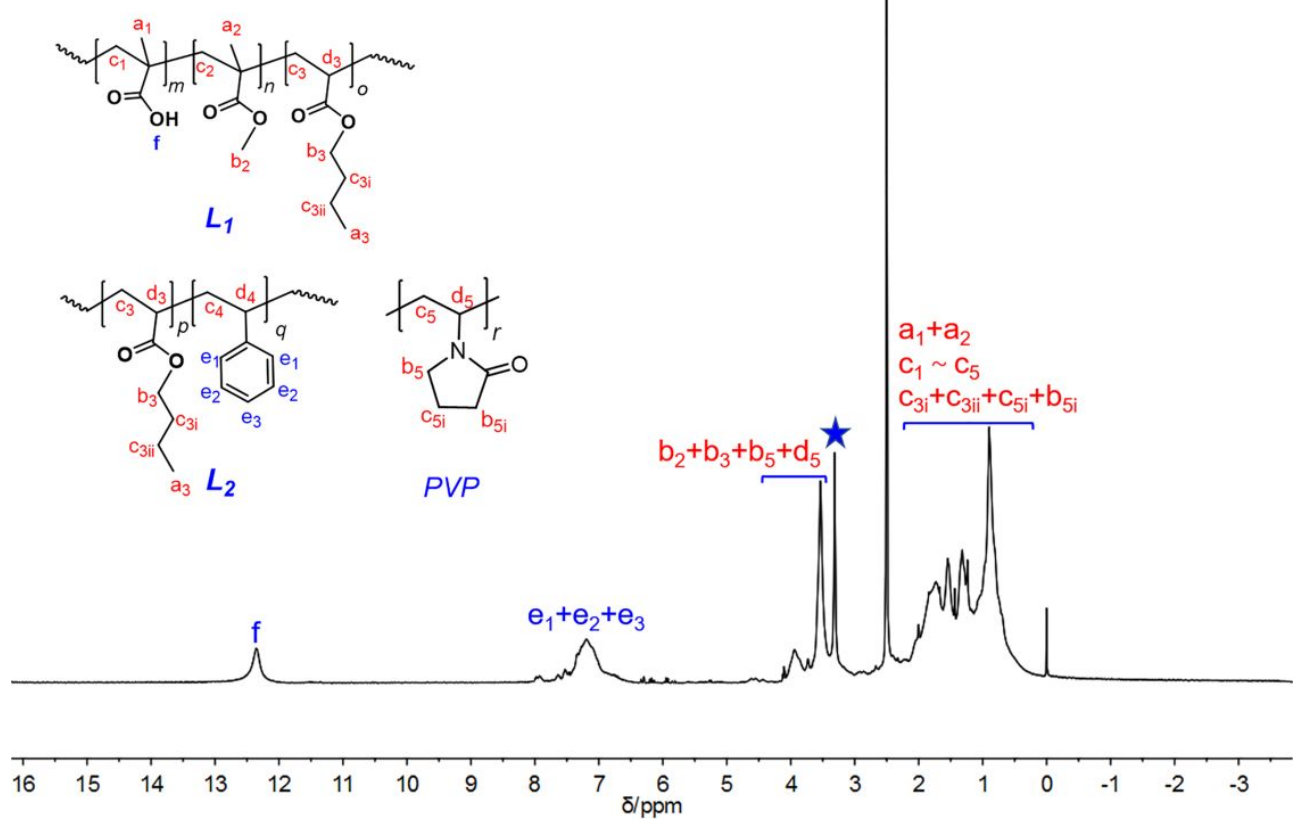

Figure S6. ${ }^{1} \mathrm{H}-\mathrm{NMR}$ spectra of StAPs-A (recorded in DMSO- $\mathrm{d}_{6}$ ). 